\title{
Impairment of proliferation and NK activity of spleen leucocytes in young rats treated with a leptin antagonist during the physiological leptin surge through postnatal days 5-10
}

\author{
M. De La Fuente ${ }^{1}$, L. Marín ${ }^{1}$, O. Hernandez ${ }^{1}$, V. Mela ${ }^{1}$, A. Gertler ${ }^{2}$ and M. P. Viveros ${ }^{1}$ \\ ${ }^{1}$ Department of Physiology. Faculty of Biology, Complutense University of Madrid and ${ }^{2}$ The Robert H. Smith Faculty of \\ Agriculture, Food and Environment, The Hebrew University of Jerusalem, Rehovot, Israel
}

\begin{abstract}
It is known that there is a physiological increase of leptin in rodents during the early postnatal (PND) period, from PND 5 to PND 10, with a peak at PND9-10, which seems to be related to brain developmental processes. We have previously shown that rats submitted to $24 \mathrm{~h}$ of maternal deprivation (MD) at PND 9 showed an impairment of several immune functions that were evident in the neonatal and adolescent periods and persisted into adulthood ${ }^{(1)}$. Since conditions with a reduced production of leptin, a very important factor in modulating immune and inflammatory reactions ${ }^{(2)}$, are associated with increased susceptibility to infection ${ }^{(3)}$, it is likely that the decrease in circulating leptin levels in MD rats is related with their impaired immune response. The aim of the present study was to determine if the use of a leptin antagonist during the period corresponding to the physiological surge of leptin (PND5-10) could affect several relevant functions of the immune cells in young rats. Young female and male rats were divided into two groups: 1) Treated with rat "mono-pegylated super active leptin antagonist" (mutant D23L/L39A/D40A/F41A), injected subcutaneously for 6 days (PND5-10) (5 mg/kg/day) (T) and 2) Injected with the corresponding vehicle for the same period (Controls: C). When males and females reached 43 days and 33 days of age, respectively, the animals were sacrificed and their spleens were removed. In the spleen leucocytes the proliferation (basal and in the response to two mitogens, namely ConA and LPS) as well as the NK activity (percentage of lysis of murine tumour cells) were analysed. The results showed that in young male and female rats treated with mono-pegylated leptin antagonist, the anti-tumour NK activity was significantly lower than in their controls counterparts $(P<0.001$ and $P<0.05$, respectively). The proliferative response of lymphocytes to ConA decreased significantly $(P<0.05)$ in the female $\mathrm{T}$ group. These results suggest that the physiological leptin surge during the neonatal PN5-PND 10 period is involved in the establishment of an adequate immune response and support the notion that the impaired immune response of MD rats is related to the MD induced leptin deficiency in a critical period for developmental (programming) leptin actions.
\end{abstract}

This work was supported by MICINN: BFU2011-30336 and BFU2009-10109, UCM (910379ENEROINN and UCM 951579), RETICEF (RD06/0013/ $0003)$ and RTA (RD06/0001/1013) from ISCIII.

1. De la Fuente M, Llorente R, Baeza I et al. (2009) Ann N Y Acad Sci 1153, 176-183.

2. Fantuzzi G (2009) Brain Behav Immun 23, 405-410.

3. Fernandez-Riejos P, Najib S, Santos-Alvarez J et al. (2010) Mediators Inflammation. Doi:10.1155/2010/568343. 